OPEN ACCESS

Edited by:

Xiaojun Chen,

Nanjing Medical University,

China

Reviewed by:

Lubin Jiang,

Institut Pasteur of Shanghai,

Chinese Academy of Sciences

(CAS), China

Fangli $L u$,

Sun Yat-Sen University,

China

*Correspondence: Yanwei Qi

qiyanwei@gzhmu.edu.cn

Xinhua Wang

xinhuaw@gzhmu.edu.cn

tThese authors have contributed equally to this work

Specialty section: This article was submitted to

Parasite and Host,

a section of the journal

Frontiers in Cellular

and Infection Microbiology

Received: 28 February 2021

Accepted: 26 April 2021

Published: 17 May 2021

Citation:

Qi Y, Zhang Y, Zheng G,

Chen B, Zhang M, Li J,

Peng T, Huang J and Wang X (2021) In Vivo and In Vitro Genome-Wide

Profiling of RNA Secondary Structures

Reveals Key Regulatory Features

in Plasmodium falciparum.

Front. Cell. Infect. Microbiol. 11:673966.

doi: 10.3389/fcimb.2021.673966

\section{In Vivo and In Vitro Genome-Wide Profiling of RNA Secondary Structures Reveals Key Regulatory Features in Plasmodium falciparum}

\author{
Yanwei ${ }^{1 *+}{ }^{1 *}$, Yuhong Zhang ${ }^{1 \dagger}$, Guixing Zheng ${ }^{2}$, Bingxia Chen ${ }^{3}$, Mengxin Zhang ${ }^{3}$, \\ Jian $L^{4}{ }^{4}$, Tao Peng ${ }^{5}$, Jun Huang ${ }^{1}$ and Xinhua Wang ${ }^{6 *}$ \\ 1 Department of Pathogenic Biology and Immunology, School of Basic Medical Sciences, Guangzhou Medical University, \\ Guangzhou, China, 2 Department of Blood Transfusion, The First Affiliated Hospital of Guangzhou Medical University, \\ Guangzhou Medical University, Guangzhou, China, ${ }^{3}$ The Third Clinical School, Guangzhou Medical University, Guangzhou, \\ China, ${ }^{4}$ State Key Laboratory of Cellular Stress Biology, Innovation Center for Cell Signaling Network, School of Life \\ Sciences, Xiamen University, Xiamen, China, ${ }^{5}$ Sino-French Hoffmann Institute, State Key Laboratory of Respiratory Disease, \\ School of Basic Medical Science, Guangzhou Medical University, Guangzhou, China, ${ }^{6}$ The First Affiliated Hospital of \\ Guangzhou Medical University, Guangzhou Medical University, Guangzhou, China
}

It is widely accepted that the structure of RNA plays important roles in a number of biological processes, such as polyadenylation, splicing, and catalytic functions. Dynamic changes in RNA structure are able to regulate the gene expression programme and can be used as a highly specific and subtle mechanism for governing cellular processes. However, the nature of most RNA secondary structures in Plasmodium falciparum has not been determined. To investigate the genome-wide RNA secondary structural features at single-nucleotide resolution in $P$. falciparum, we applied a novel high-throughput method utilizing the chemical modification of RNA structures to characterize these structures. Structural data from parasites are in close agreement with the known 18S ribosomal RNA secondary structures of $P$. falciparum and can help to predict the in vivo RNA secondary structure of a total of 3,396 transcripts in the ring-stage and trophozoite-stage developmental cycles. By parallel analysis of RNA structures in vivo and in vitro during the Plasmodium parasite ring-stage and trophozoite-stage intraerythrocytic developmental cycles, we identified some key regulatory features. Recent studies have established that the RNA structure is a ubiquitous and fundamental regulator of gene expression. Our study indicate that there is a critical connection between RNA secondary structure and mRNA abundance during the complex biological programme of $P$. falciparum. This work presents a useful framework and important results, which may facilitate further research investigating the interactions between RNA secondary structure and the complex biological programme in $P$. falciparum. The RNA secondary structure characterized in this study has potential applications and important implications regarding the identification of RNA structural elements, which are important for parasite infection and elucidating host-parasite interactions and parasites in the environment. 


\section{INTRODUCTION}

RNA, a multitasking biomolecule, plays important roles in many aspects of cellular and physiological processes, such as the regulation of transcription, RNA processing and stability including splicing events, and translation (Wan et al., 2011; Mortimer et al., 2014). As the carrier of genetic information for translation into proteins, RNA molecules can fold into a wide array of complicated and exquisite secondary and tertiary structures via complex patterns of intramolecular base pairing formed by Watson-Crick-base pairing (Vandivier et al., 2016). These complex secondary and tertiary structures of RNAs play important roles in regulating such processes as catalytic and ligand sensing, the regulation of mRNA maturation, translation and turnover, alternative polyadenylation and alternative splicing (Ding et al., 2014).

Due to the multiple functions of RNA structural elements changed by base pairing and folding, they can be considered another layer of the genetic code that is only beginning to be understood (Bevilacqua et al., 2016). Characterizing these complex global RNA structures, especially in vivo, is essential to achieve a mechanistic understanding of the function and regulation of RNA transcripts. However, it remains challenging to understand the role and mechanism of mRNA secondary structure-based regulation, especially the long-standing challenges in RNA structure modelling. Although structural studies can examine global patterns of RNA structures in a single sample, these RNA structures are not static in vivo. At the same time, RNA structure can be refolded by RNA binding proteins or have posttranscriptional covalent modifications that result in drastic changes in secondary structure in response to changes in the prevailing physicochemical environment of the cell and the effects of various stimuli (Vandivier et al., 2016). These studies may help to identify additional temperature sensors (RNA thermometers), enzymes (ribozymes), ligandbinding sensors (riboswitches) or other environmentally responsive structural elements in various organisms, although not in Plasmodium parasites.

Investigation of RNA secondary structures associated with specific biological events is therefore essential to understanding the functions and roles of these RNA molecules. The secondary structure of RNA is relatively stable and is present across the length of an mRNA, including the CDS and UTRs (Mignone et al., 2002). A number of studies using global RNA structure-probing approaches have explored the RNA structurome in different species, such as mouse cell lines (Spitale et al., 2015), yeast (Kertesz, 2010; Talkish et al., 2014), human cells (Wan et al., 2014), Arabidopsis thaliana (Zheng et al., 2010; Li et al., 2012b; Ding et al., 2014; Incarnato et al., 2014), Drosophila melanogaster and Caenorhabditis elegans (Li et al., 2012a), zebrafish (Kaushik et al., 2018), Yersinia pseudotuberculosis (Righetti et al., 2016), and, recently Oryza sativa (Su et al., 2018). However, a comprehensive whole-genome analysis of RNA secondary structures has not been obtained for Plasmodium parasites. Furthermore, a thorough analysis of secondary structure correlations between different developmental stages has never been accomplished. In this study, we applied the novel biochemical approach icSHAPE which, to the best of our knowledge, enables the first global view of RNA secondary structures in the major human pathogen Plasmodium falciparum for all four bases in vivo and in vitro at the ring stage and trophozoite stage of parasite development.

Malaria is caused by any one of five species of protozoan parasites, namely, $P$. falciparum, $P$. vivax, $P$. malariae, $P$. ovale, and $P$. knowlesi, and is one of the most important tropical parasitic diseases with high morbidity and mortality rates, especially in many developing countries. In 2019, approximately 229 millions cases of malaria and 409,000 deaths were reported in endemic countries each year, especially in the sub-Saharan African region (World Health Organization, 2020). Various measures have been employed to control the disease, including vector control, bed-nets, and chemotherapy, but these methods have achieved only limited success (Choi et al., 2019). Given the lack of effective vaccines, the widespread resistance to anti-malaria drugs in current use (Capela et al., 2019), and poorly understood molecular mechanisms, it has become an urgently important to identify and develop new effective strategies to control malaria.

The major strength of this study is that it explained the landscape of the RNA secondary structure associated with parasite development at single-nucleotide resolution. Our analysis profiled the structure of more than 3,396 (in vivo) and 2,024 (in vitro) transcripts in the ring-stage and trophozoite-stage developmental cycles. In this study, we showed that (1) RNA secondary structures in $P$. falciparum have common characteristics similar to those of other diverse organisms, such as UTR regions, CDS regions, noncoding RNAs, and malaria mitochondrion. (2) Structural changes are dramatically different in the ring and trophozoite stages but less pronounced in the ring stage than in the trophozoite stage. (3) Comparing the in vivo and in vitro structures indicates the important role of RNA-binding protein (RBP) in structure formation. (4) Combined with transcriptome data, RNA secondary structure changes were significantly associated with transcriptome changes. Therefore, our results make it possible to obtain a framework for understanding how malaria parasites develop through changes in RNA structure in vivo and potential use for further investigations. Our results imply that there is a critical connection between the RNA secondary structure and the complex biological programme of Plasmodium falciparum, but the mechanism governing this process needs to be further elucidated.

\section{MATERIALS AND METHODS}

\section{Parasite Culture and Synchronized}

The P. falciparum strain 3D7 was cultured in human $\mathrm{O}^{+}$ erythrocytes at $5 \%$ hematocrit under standard in vitro conditions as previously described (Trager and Jensen, 1976). Cultures were synchronized twice at ring stage with 5\% D-sorbitol treatments performed 8 hours apart (Lambros and Vanderberg, 1979). The cultures were expanded to accommodate harvesting of at least $200 \mathrm{~mL}$ of culture at each planned time-point. Cultures $(\sim 8 \%$ parasitemia in $5 \%$ hematocrit) were harvested 48 hours (ring stage) and 72 hours (trophozoite stage) after the first sorbitol 
treatment. Next, the cells were harvested at $2000 \mathrm{rpm}$ for $3 \mathrm{~min}$ to obtain packed RBCs. The packed RBCs were lysed by a $0.05 \%$ saponin solution, and parasite pellets were washed twice using phosphate-buffered saline ( $\mathrm{pH}$ 7.4). The remaining pellets were used for icSHAPE library construction, and total RNA was extracted directly, or conversely, parasites were stored in $10 \mathrm{~mL}$ of TRIzol reagent at $-80^{\circ} \mathrm{C}$ prior to RNA extraction.

\section{In Vivo and In Vitro NAI- $\mathrm{N}_{3}$ Chemical Probing and RNA Sample Extraction}

For in vivo $\mathrm{NAI}_{3} \mathrm{~N}_{3}$ modification of parasite RNAs, the washed parasites pellets were treated as described previously (Flynn et al., 2016) before total RNA extraction. The parasites pellets were equally divided into three parts, one part for $\mathrm{NAI}-\mathrm{N}_{3}$ in vivo modification and the two other parts for the DMSO control. NAI$\mathrm{N}_{3}$ concentration/times were chosen as in our experiment described in the following section to provide a similar overall level of modification for samples in vivo. Briefly, almost all PBS was removed from the pelleted parasite, the parasite pellet was resuspended in $200 \mu \mathrm{l}$ of $100 \mathrm{mM} \mathrm{NAI-N_{3 }}$ solution or $200 \mu \mathrm{l}$ of DMSO solution, and the suspension was immediately mixed by inversion and incubated at $37^{\circ} \mathrm{C}$ with end-over-end rotation for $15 \mathrm{~min}$. Before use, the $100 \mathrm{mM} \mathrm{NAI-N_{3 }}$ was thawed completely to $37^{\circ} \mathrm{C}$. The reaction was stopped by quickly placing the pellets on ice and collecting them by centrifugation at $14,000 \mathrm{~g}$ at $4^{\circ} \mathrm{C}$ for $30 \mathrm{~s}$. Next, the supernatants were removed, and the pellets were transferred to $15-\mathrm{ml}$ tubes and resuspended in $10 \mathrm{ml}$ of prewarmed TRIzol Reagent. Total RNA was isolated as recommended by the manufacturer's protocol followed by phenol/chloroform extraction and ethanol precipitation. The RNA yield was measured using a Nanodrop spectrophotometer, and RNA quality was assessed by $1 \%$ agarose gel analysis. We ensured that clear $18 \mathrm{~S}$ and $28 \mathrm{~S}$ rRNA bands were present. We needed to construct total RNA/polyA-selected RNA in vivo SHAPE RNA libraries. In this study, polyA mRNA was obtained using the NEBNext ${ }^{\circledR}$ Poly-A mRNA Magnetic Isolation Module (NEB).

For in vitro $\mathrm{NAI}-\mathrm{N}_{3}$ probing, we also needed to construct total RNA/ribosomal in vitro SHAPE RNA libraries. For total RNA/ribosomal in vitro SHAPE RNA libraries, no additional processing was needed. For polyA-selected in vitro SHAPE libraries, polyA selection was performed first using polyT oligomagnetic beads before $\mathrm{NAI}-\mathrm{N}_{3}$ in vitro modification was undertaken. Heat-denatured total RNA or polyA+ RNA samples were obtained from DMSO-treated pellets at $95^{\circ} \mathrm{C}$ for $2 \mathrm{~min}$ and were later transferred to ice to cool. The NAI-N $\mathrm{N}_{3}$ final concentration and times for in vitro modification were $100 \mathrm{mM}$ and $37^{\circ} \mathrm{C}$ for $10 \mathrm{~min}$. The reactions were stopped by moving the samples to ice, and the samples were purified using a Zymo RNA Clean \& Concentrator-5 column according to the manufacturer's protocol.

\section{Quantification of Modifications by Primer Extension and Resolution by Capillary Electrophoresis}

Incubation time and NAI-N ${ }_{3}$ concentration can strongly affect single-hit kinetics; therefore, we focused on optimizing these two parameters, although it is possible that other factors, such as temperature and buffer conditions, could also affect these kinetics (Ding et al., 2015). We took a time course (5 min, $10 \mathrm{~min}, 15 \mathrm{~min}$ and $30 \mathrm{~min}$ ) of in vivo NAI-N $\mathrm{N}_{3}$ modification as an example for a region of $18 \mathrm{~S}$ rRNA in $P$. falciparum by capillary electrophoresis for at least three times. Seven micrograms of total RNA (non-polyA-selected) was treated with DNase (NEB) to remove residual genomic DNA in the RNA sample. Next, the DNA-free total RNA was reversetranscribed using SuperScript III (Invitrogen) by a colourcoded fluorescently FAM-labelled oligonucleotide in the 271-bp-293-bp region of the $18 \mathrm{~S}$ rRNA gene. The PCR programme was as follows: samples were heated to $65^{\circ} \mathrm{C}$ for $5 \mathrm{~min}$, maintained on ice for at least $2 \mathrm{~min}$, and incubated at $50^{\circ} \mathrm{C}$ for $1 \mathrm{~h}$ followed by $95^{\circ} \mathrm{C}$ for $5 \mathrm{~min}$, and the samples were maintained at $4^{\circ} \mathrm{C}$ after the reaction ended. The reversetranscribed cDNA products were electrophoresed using an ABI 3730XL DNA Sequencer. The results were presented by GeneMarker (V2.7.0, SoftGenetics, LLC.)

\section{Determination of Fragmentation Time by Primer Extension Electropherograms}

Total RNA was extracted from parasite lysates without any chemical reagent modification. In a $20-\mu \mathrm{L}$ reaction, $\sim 2 \mu \mathrm{g}$ of total RNA was fragmented using RNA fragmentation reagents (Ambion) for the proper time at $70^{\circ} \mathrm{C}$. After the end of the experiment, $10 \times$ stop solution was added to each sample, and the samples were immediately placed on ice. Next, fragmented RNA was purified with a Zymo RNA Clean \& Concentrator-5 column. The purified fragmented RNA was reverse-transcribed by primer extension with gene-specific colour-coded fluorescently FAMlabelled oligonucleotides (5'-ACCCTAACATCAAAAGCT GATAGG-3'), as described above. The length of every fragment was assessed using an ABI 3730XL DNA Sequencer (Applied Biosystems). The results were shown by GeneMarker (V2.7.0, SoftGenetics, LLC.).

\section{RNA-Seq Illumina Library Construction}

For RNA-seq library construction, the NEBNext Ultra Directional RNA Library Prep Kit (NEB) was used to prepare a validated strand-specific, polyA-selected RNA sequencing library according to the manufacturer's instructions in the kit. Briefly, mRNA was purified from $3 \mu \mathrm{g}$ of total RNA using the Poly(A) mRNA Magnetic Isolation Module. Fragmentation was carried out at $94^{\circ} \mathrm{C}$ for 7 min in NEBNext First Strand Synthesis Reaction Buffer. First strand cDNA was synthesized using random primers and ProtoScript II Reverse Transcriptase (with $0.1 \mu \mathrm{g} / \mu \mathrm{l}$ actinomycin $\mathrm{D}$ and murine RNase inhibitor). Second strand cDNA was synthesized using Second Strand Synthesis Enzyme Mix and incubated at $16^{\circ} \mathrm{C}$ for $1 \mathrm{~h}$. Next, the double-stranded cDNA was purified using 1.8X Agencourt AMPure XP Beads. End prep of a cDNA library was performed by NEBNext End Prep Enzyme Mix at $20^{\circ} \mathrm{C}$ for $30 \mathrm{~min}$ followed by $65^{\circ} \mathrm{C}$ for $30 \mathrm{~min}$. NEBNext adaptors were ligated to the cDNAs with the function of Blunt/TA Ligase Master Mix at $20^{\circ} \mathrm{C}$ for $15 \mathrm{~min}$, and fragments measuring 150-200 bp were purified 
using AMPure XP beads. The samples were later treated with $3 \mu \mathrm{l}$ NEBNext USER Enzyme at $37^{\circ} \mathrm{C}$ for 15 min before PCR amplification. Library amplification was performed using NEBNext Q5 Hot Start HiFi PCR Master Mix, NEBNext Universal PCR Primer for Illumina, and Index (X) Primer. The primer and oligonucleotide sequences used in the experiment are shown in Supplementary Table 1. The PCR machine was programmed as follows: initial denaturation at $98^{\circ} \mathrm{C}$ for $30 \mathrm{~s}$, denaturation at $98^{\circ} \mathrm{C}$ for $10 \mathrm{~s}$ and annealing/extension at $65^{\circ} \mathrm{C}$ for $75 \mathrm{~s}$ for $12 \mathrm{cycles}$. The end step was final extension at $65^{\circ} \mathrm{C}$ for 5 min. The PCR products were purified using an AMPure XP system before the quality of the libraries was assessed using an Agilent Bioanalyzer 2100, and the libraries were sequenced on an Illumina HiSeq 2000 at Vazyme Biotech Co., Ltd. (Nanjing, China) and GENEWIZ company (Suzhou, China). Paired-end reads were mapped to the $P$. falciparum 3D7 assembly release-33 downloaded from PlasmoDB (http://plasmodb.org/common/ downloads/Current_Release/Pfalciparum3D7/). Next, read counts were collected, and gene expression levels among different samples were estimated.

\section{icSHAPE Deep-Sequencing Illumina Library Construction}

For the icSHAPE library, we used the methods developed by Ryan A Flynn et al. as described previously (Flynn et al., 2016), with some modifications. For total RNA/ribosomal RNA libraries, no additional processing was needed. For polyAselected libraries, polyA selection was performed first using polyT oligo magnetic beads. Briefly, DNA-free mRNA, which was purified from total RNA using polyT oligo magnetic beads, was subjected to a biotin click reaction with the modification reagent $\mathrm{NAI}-\mathrm{N}_{3}$ at $37^{\circ} \mathrm{C}$ in a thermomixer for $2 \mathrm{~h}$ at 1,000 r.p.m. with $4 \mathrm{U} / \mu \mathrm{l}$ RiboLock RNase inhibitor. Fragmentation was performed using RNA fragmentation reagent (Ambion) at $70^{\circ} \mathrm{C}$ for $3 \mathrm{~min}$. Next, purified fragmented RNA was end-repaired by T4 polynucleotide kinase (NEB) and incubated at $37^{\circ} \mathrm{C}$ for $1 \mathrm{~h}$. A $3^{\prime}$ biotin RNA linker or a 3'-ddC RNA linker were ligated to the endrepaired RNA samples by T4 RNA ligase 1 . After each above step, the reaction was purified by a Zymo RNA Clean \& Concentrator-5 column according to the manufacturer's protocol.

First strand cDNA was subsequently synthesized using $1 \mu \mathrm{l}$ of $1 \mu \mathrm{M}$ RT primer (with a 4-nt barcode for separate samples in one sequencing lane). After reverse transcription, isolation by MyOneC1 streptavidin beads and size selection (>70 nt) of NAI-N ${ }_{3}$-modified molecules from cDNA products, the concentrated cDNA samples were purified with a Zymo DNA Clean \& Concentrator-5 column. The size-selected cDNA was circularized by $5 \mathrm{U} / \mu \mathrm{l}$ CircLigase II (Epicentre) and incubated at $60^{\circ} \mathrm{C}$ for $2 \mathrm{~h}$. Amplification was performed using NEBNext High-Fidelity 2X PCR Master Mix, Solexa-P5 primer, and Solexa-P3 primer. The $\mathrm{qPCR}$ machine was programmed as follows: $98^{\circ} \mathrm{C}$ for $45 \mathrm{~s}, 98^{\circ} \mathrm{C}$ for $15 \mathrm{~s}, 65^{\circ} \mathrm{C}$ for $20 \mathrm{~s}$, and $72^{\circ} \mathrm{C}$ for $30 \mathrm{~s}$. For the in vivo and in vitro NAI- $\mathrm{N}_{3}$-treated samples, 14 cycles were employed, and for DMSO-treated samples, 11 cycles were employed. Finally, the PCR-amplified library was gel sizeselected using 10\% TBE PAGE to obtain a size between $200 \mathrm{bp}$ and $300 \mathrm{bp}$. The primer and oligonucleotide sequences used in the experiment are shown in Supplementary Table 1.

Final icSHAPE library material was quantified on a Bioanalyzer High Sensitivity DNA Chip 2100 (Agilent) and Qubit and was sent for deep sequencing and Illumina HiSeq analysis at Vazyme Biotech Co., Ltd. (Nanjing, China) and GENEWIZ Company (Suzhou, China).

\section{Sequencing Data Analysis}

The quality of all PF data from Illumina's HiSeq sequencer was assessed by FastQC (Babraham Bioinformatics), and the percentages of reads with Q20, Q30, and GC content were calculated. All sequenced libraries were collapsed to remove PCR duplicates, reads containing adapters, reads containing polyN, and low-quality scores.

All downstream analyses were based on clean data with barcodes ( 1 -13 bases in each read) removed by using Trimmomatic (Bolger et al., 2014), and reads with bases $<35$ nt were discarded after barcode removal. All the clipped sequenced libraries were later mapped to the $P$. falciparum genome and transcriptome (build release-33 downloaded from PlasmoDB on June 26th, 2017 http://plasmodb.org/common/ downloads/Current_Release/Pfalciparum3D7/) using bowtie (Langmead et al., 2009) implemented with mismatch $=0$ (bases from 1-28 nt) and default parameters. The ' -1 positions' of each sequencing read represented the reverse transcription stop, which corresponded to modified nucleotides in the NAI-N group and intrinsic/fragmentation modified in the DMSO control group. The number of times a base was mapped as ' -1 positions' was counted by Perl scripts and bed tools (Quinlan and Hall, 2010). All icSHAPE profiles of all mapped RNA species were sorted into different files by chromosome for the two experiments at the two developmental stages at the ring stage and trophozoite stage. All reads mapped to the genome and transcriptome from different libraries were normalized by the total number of RT stops in each library [NAI-N $_{3}$ in vivo, NAI$\mathrm{N}_{3}$ in vitro, and DMSO control] and sequencing depth. The icSHAPE signals/scores for each RNA position were calculated as the ratio of $\mathrm{NAI}-\mathrm{N}_{3} / \mathrm{DMSO}$ numbers of modified nucleotides after all reverse transcription stops were normalized by the amount of all reads in each library (Talkish et al., 2014). To reduce the potential overestimation of structural signals of bases with low/zero coverage, a small number 5 was added to the numbers of modified nucleotides, which were normalized by sequencing depth (Wan et al., 2014). The final analysis of the icSHAPE sums was performed with Microsoft Excel 2013 and Student's t-tests using R.

\section{Computation of the RNA Secondary Structures With icSHAPE Scores}

An increasing number of online web services or software can be used for the prediction of RNA secondary structure (Jin et al., 2011). In our research, colour coding by icSHAPE signalling was performed using online ViennaRNA Web Services (http://rna. tbi.univie.ac.at/) with five different colours. Specifically, the coloured base pair was placed into the known regions of the 
$18 \mathrm{~S}$ rRNA secondary structure, and the experimental single/double results were subsequently checked against the known ways of pairing. For the RNA secondary structure profile, all selected secondary structure models were generated using RNAstructure 6.112 on a Windows operating system (http://rna.urmc.rochester.edu/RNAstructure.html) with and without hard constraints due to the extracted icSHAPE score profiles of all single RNAs that were calculated (After testing and comparing on the known regions of $18 \mathrm{~S}$ rRNA, we concluded that in RNAstructure software, the parameter of threshold for force single stranded was 1.5, and the threshold for chemical modification was 1.0, for our icSHAPE scores profiles.) In our approach, the nucleotides that have icSHAPE scores above a specified threshold (1.5) are forced to be single-stranded. Otherwise, the positions are treated as being inaccessible to chemical modification, i.e., double-stranded or crowded by specific protein. We also used the current best parameters (read shape reactivity - pseudo energy constraints with slope $=$ 1.8 , intercept $=-0.613$ ) for icSHAPE-directed RNA structure prediction. Alternatively, webserver tools were also used at http://rna.urmc.rochester.edu/RNAstructureWeb/. RNA structures were drawn and coloured using StructureEditor.

\section{RESULTS}

\section{Determination of the Conditions for Single-Hit Kinetics and Fragmentation Time by Primer Extension Electropherograms}

To obtain the single-nucleotide resolution and genome-wide RNA secondary structural features in P. falciparum, NAI-N 3 should be titrated to single-hit kinetics in structure probing. In our pilot experiments, we used capillary electrophoresis (CE) (Vasa et al., 2008), which has extended the amenable length to $500 \mathrm{nt}$, to confirm the NAI-N $\mathrm{N}_{3}$-labelled $18 \mathrm{~S} 5$ ' end rRNA structure and compare those structures with the known secondary structure of PF 18 S rRNAs (Wong et al., 2014) (Supplementary Figure 1). Our results showed that we obtained nearly identical secondary structure for the 5 ' end of $18 \mathrm{~S}$ rRNA as previously reported (Supplementary Figure 1). These results show that the 15 -min time point is the optimal duration for NAI-N $\mathrm{N}_{3}$ modification, as it is the longest time point for which single-hit kinetics still occur as revealed by consistency with the known structure. The 30 -min time point is too long, as revealed by the significant of loss modified points and the increase of shorter length bands. Next, we used this condition for all the following in vivo RNA modifications. IcSHAPE in vivo libraries were subsequently prepared using RNA from pellets treated with $100 \mathrm{mM} \mathrm{NAI}^{-\mathrm{N}_{3}}$ for $15 \mathrm{~min}$, and control pellets not treated with NAI-N $\mathrm{N}_{3}$ but with DMSO solution.

Appropriate fragment sizes have a critical role in ensuring the highest signal to-noise ratio and are need for efficient obtaining meaningful reads and modification sites via RT-PCR and sequencing. Short RNAs (measuring 100 nt) are the most common strategies that have been optimized to achieve single- hit kinetics of chemical modification, which can result in $\sim 10 \%$ of all transcriptions being structurally informative (Merino et al., 2005). For this purpose, we first determined the fragmentation time by primer extension electropherogram experiments (Supplementary Figure 2). The results showed that the 3-min time point was the optimal fragment time for obtaining $\sim 100 \mathrm{nt}$ short RNAs. The 4-min time point is too long, as revealed by significant loss of $\sim 100 \mathrm{nt}$ short RNAs.

\section{Comparison of icSHAPE Data to Known RNA Structures}

To evaluate the accuracy of our $\mathrm{NAI}_{-} \mathrm{N}_{3}$-labelledicSHAPE approach, we first used capillary electrophoresis-based probing to compare $\mathrm{NAI}-\mathrm{N}_{3}$ modifications from icSHAPE with those from conventional gel-based modification read-outs. These results indicated strong agreement for the special regions of $18 \mathrm{~S}$ rRNA tested (Figures 1A, B). Second, we mapped our icSHAPE scores to well-studied RNA molecules (Figure 1C and Supplementary Figure 3). The secondary structure of $P$. falciparum A-type 18S small subunit ribosomal RNA (http:// www.rna.ccbb.utexas.edu) has been published (Cannone et al., 2002); therefore, we used this known RNA secondary structure as a model to compare with our icSHAPE modification RNA secondary structure according to our icSHAPE scores. In vitro RNA icSHAPE libraries prepared using RNA from in vitro NAI$\mathrm{N}_{3}$ modification with no ribosomal removal were sequenced to produce an average of 14.5 million reads in the 2092-bp region of $P$. falciparum A-type $18 \mathrm{~S}$ small subunit ribosomal RNA. The icSHAPE scores for each site on $18 \mathrm{~S}$ rRNA are depicted in Supplementary Table 2 . Colour coding by icSHAPE signal was performed using online ViennaRNA Web Services (http://rna. tbi.univie.ac.at/) with five different colours according to the icSHAPE scores generated from icSHAPE-seq (icSHAPE scores $<1.5$ were marked in red; icSHAPE scores $1.5-2.0$ were marked in yellow; icSHAPE scores 2.0-2.5 were marked in green; icSHAPE scores 2.5-3.0 were marked in blue; icSHAPE scores $>3.0$ were marked in purple). The coloured base pair was placed into the known regions of the $18 \mathrm{~S}$ rRNA secondary structure, and the experimental single/double results were subsequently checked against the known ways of pairing.

In the known secondary RNA structure of entire 18S A-type rRNA (length of 2,092 nt, located on chromosome 5), approximately $41.0 \%$ of bases are single-stranded, $55.2 \%$ of bases are double-stranded, and 3.8\% (80 nt) are indeterminate. In the entire $18 \mathrm{~S}$ A-type rRNA, 43.9\% (the icSHAPE score is above 1.5 , indicating that this position is single-stranded) of the bases that show high in vivo icSHAPE scores (defined as 1.5) in our data set correspond to single-stranded regions in the phylogenetic structure (41.0\%), whereas $56.1 \%$ (the icSHAPE score is below 1.5, indicating that this position is a double strand) of the bases that show low in vivo icSHAPE scores in our dataset correspond to base-paired regions in the phylogenetic structure (55.2\%) and presumably are protected by either ribosomal proteins or non-canonical base-pairing tertiary RNA structures. Our data showed that there is a high correlation between icSHAPE scores and the known 18S A-type RNA 
A

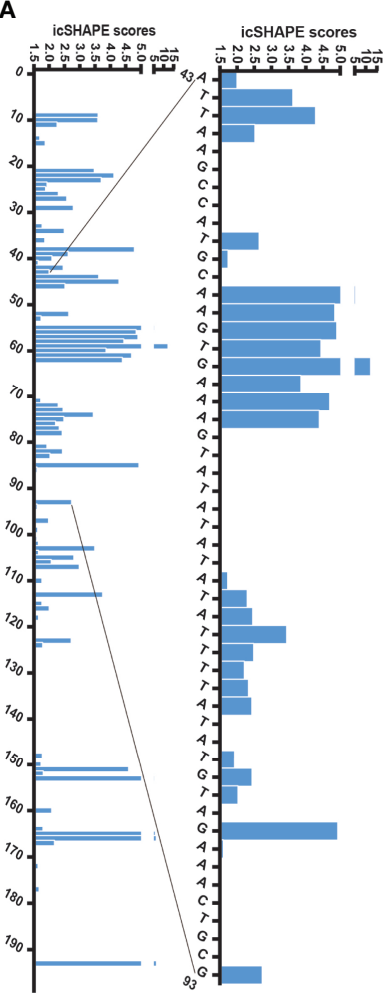

B

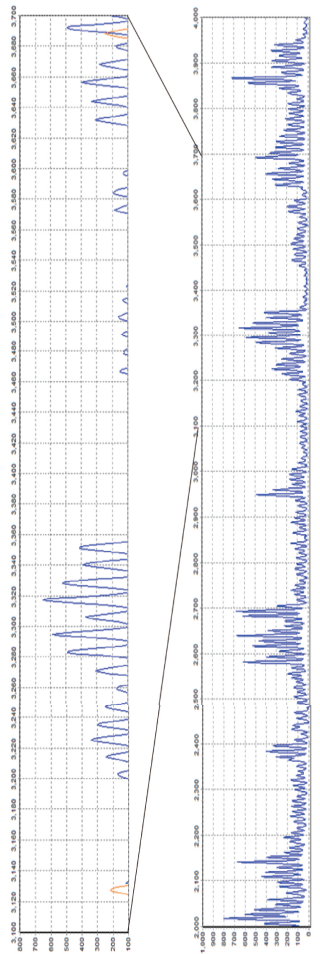

C
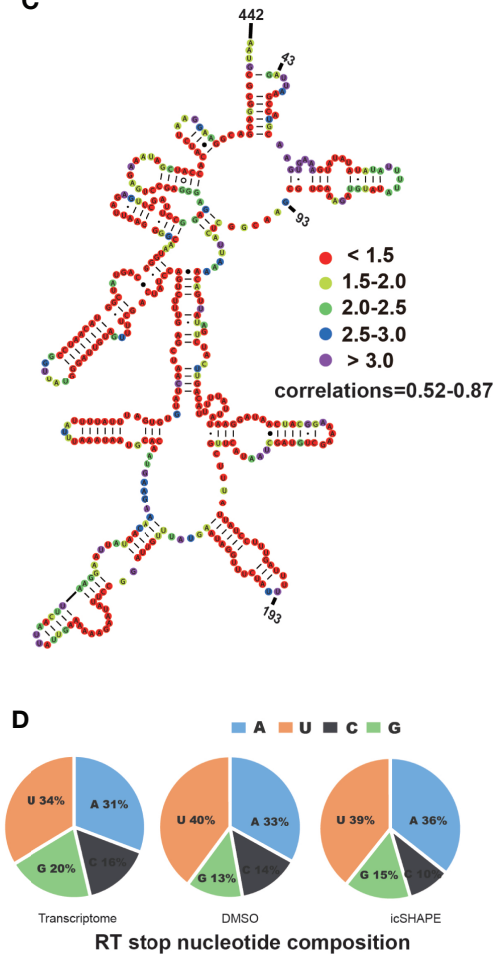

FIGURE 1 | icSHAPE scores accurately maps the region 43-442bp of P. falciparum 18S small subunit rRNA (blood stage) icSHAPE scores accurately maps the region 43-442bp of Plasmodium falciparum 18S small subunit ribosomal RNA (blood stage) and agrees with capillary electrophoresis-based in vivo structure probing. (A) icSHAPE scores for nucleotides 1-193 is shown on the left. The sites of nucleotides which showed have the scores above 1.5, this score means this position is single-strand. (B) The gel from 18S A-type rRNA NAI- $\mathrm{N}_{3}$ modification read-out by capillary electrophoresis-based probing, which was done here near the 5' end (100bp-300bp), which consist with the region on the 1-193 of the 18S rRNA. (C) Nucleotides 43-442 of the 18S A-type small subunit ribosomal RNA, colourcoded according to icSHAPE scores. The whole secondary structure of $18 \mathrm{~S}$ A-type small subunit ribosomal RNA are showed in Supplementary Figure 3. (D) Reverse transcription stop distribution for the transcriptome of 18S A-type rRNA on chromosome 5, DMSO-treated RNA control libraries or icSHAPE-treated RNAs libraries in the same regions of $18 \mathrm{~S}$ rRNA. All of the three stop distributions are highly concordant, icSHAPE in vivo treatment no enriches for any sequencing reads mapping to four bases compared to untreated control, except a slight enrichment in $\mathrm{NAI}^{-\mathrm{N}_{3}}$ library for As and Us.

secondary structure. We set the sites with icSHAPE scores above 1.5 to be localized to regions of single-stranded RNA and were consistent with traditional primer extension analysis and known RNA secondary structures. NAI-N 3 modification has no specific RT stops with almost equal numbers of four bases, consistent with the known absent or minor (Spitale et al., 2015) (with a very slight enrichment in NAI-N $\mathrm{N}_{3}$ samples for As and Us) base-

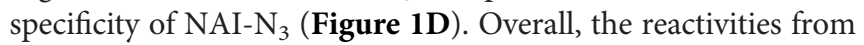
our icSHAPE libraries are consistent with the known structural mapping of $18 \mathrm{~S}$ rRNA, which is the closest in vivo model.

\section{Overview of the $P$. falciparum RNA Structurome}

To obtain a genome-wide profile of the single-nucleotide resolution RNA secondary structure during the intraerythrocytic developmental cycle of $P$. falciparum, we performed icSHAPE, as depicted in Supplementary Figure 4. P. falciparum 3D7 synchronized twice with $5 \% \mathrm{D}$-sorbitol was cultured in a $37^{\circ} \mathrm{C}$ incubator to keep the majority of the parasite population at the same stage. Smears were made by Giemsa staining and used for microscopic observation of parasite morphology at each time point.

The transcripts, with reverse transcription stop coverage no less than 2 and background base density higher than 200 as described previously (Spitale et al., 2015), were analysed. The detailed data in the icSHAPE libraries and RNA-seq libraries are displayed in Supplementary Table 3. We obtained an average of $55.38 \%$ mappability and 78.3 million qualified fragments per sample in the icSHAPE library (Supplementary Table 3). The results are highly reproducible across two biological replicates.

Finally, 2,044 and 1,701 valid structural profiles for transcripts $(35.2 \%$ and $29.4 \%$ of the ratio of all transcripts respectively) were obtained from in vivo and in vitro $37^{\circ} \mathrm{C}$ treated polyA-selected RNA ring stage libraries, among which the majority were mRNAs as shown in Figures 2A-C (and also in Supplementary Table 4). In addition 1,374 and 329 valid structural profiles for transcripts $(23.7 \%$ and $5.69 \%$ of the ratio of all transcripts respectively, with reverse transcription stop coverage no less than 2) were obtained from in vivo and 
A

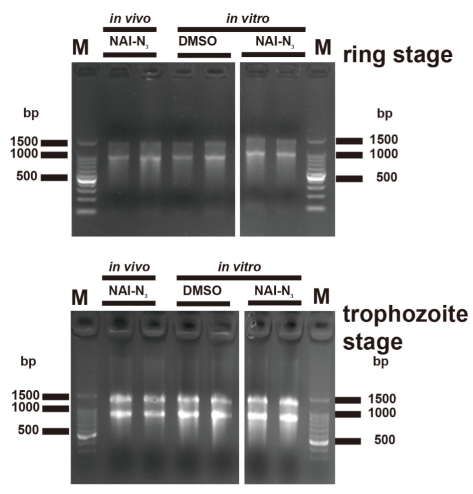

B

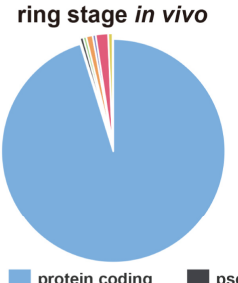

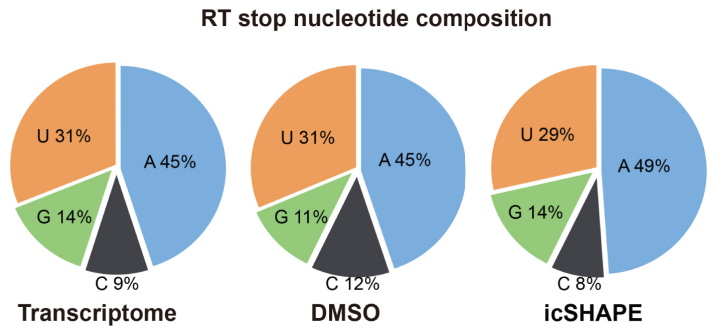

protein coding $\square$ pseudogene processed transcript

ring stage in vitro

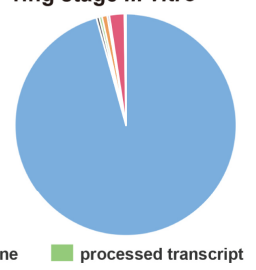

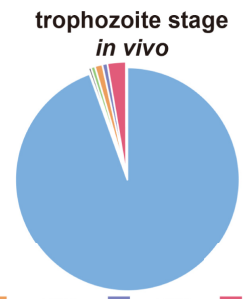

trophozoite stage

snoRNA snRNA mitochondrial apicoplast

C

\begin{tabular}{lcccccccc}
\hline & protein coding pseudogene processed transcript snoRNA & snRNA & mitochondrial & apicoplast & Total \\
\hline ring stage in vivo & 1947 & 10 & 9 & 19 & 9 & 37 & 13 & 2044 \\
ring stage in vitro & 1628 & 7 & 7 & 13 & 5 & 37 & 4 & 1701 \\
trophozoite stage in vivo & 1299 & 5 & 8 & 15 & 10 & 37 & 0 & 1374 \\
trophozoite stage in vitro & 286 & 1 & 0 & 7 & 0 & 35 & 0 & 329 \\
\hline
\end{tabular}

FIGURE 2 | icSHAPE is capable of measuring the RNA structure profiles of thousands of RNAs simultaneously in vivo and in vitro. icSHAPE is capable of measuring the RNA structure profiles of thousands of RNAs simultaneously in vivo. (A) Evaluation of RNA quality of two biological replicates between stages and conditions in vivo modification, in vitro modification, and DMSO control samples in agarose gel. (B, C) The total number of structural profiles for transcripts was classified into different classes of RNAs from a total number of 2044 transcripts for the ring stage library at 37 degree in vivo, 1701 transcripts for the ring stage library at 37 degree in vitro, 1374 transcripts for the trophozoite stage library at 37 degree in vivo, and 329 transcripts for the trophozoite stage library at 37 degree in vitro. This figure combines results from both two biological replicates. (D) Reverse transcription stop distribution for the whole transcriptome, DMSO-treated RNA control libraries or icSHAPE-treated RNAs libraries. All of the three stop distributions are highly concordant, icSHAPE in vivo treatment no enriches for any sequencing reads mapping to four bases compared to untreated control, except a slight enrichment in NAI- $\mathrm{N}_{3}$ library for As.

in vitro $37^{\circ} \mathrm{C}$ treated polyA-selected RNA trophozoite stage libraries (Supplementary Table 4). All of the three reverse transcription stop distributions for the whole transcriptome, DMSO-treated RNA control libraries or icSHAPE-treated RNAs libraries were highly concordant. The icSHAPE in vivo treatment did not enrich any sequencing reads mapping to four bases compared to the untreated control, except for a slight enrichment in the NAI-N ${ }_{3}$ library for As (Figure 2D).

While polyA purification was undertaken in those polyAselected RNA libraries before library construction, it strongly reduced the reads mapped to the tRNAs and rRNAs. We mapped the structural profiles by icSHAPE score data in vivo and in vitro from selected regions of 110 mRNAs (the top 110 structural profiles for transcripts that have the highest reverse transcription stop coverage, Supplementary Table 5) across parasites that have 5' untranslated regions (UTRs) and 3' UTR regions longer than 200 nt: 5' UTR (200 nt upstream of the start codon); coding sequence region (CDS region, $110 \mathrm{nt}$ downstream of the start codon and $110 \mathrm{nt}$ upstream of the stop codon); and 3' UTR (100 nt downstream of the stop codon) (Kertesz, 2010). Next, we used the obtained structural profiles by icSHAPE scores to investigate three global properties of parasite transcripts to evaluate the hypothesis that robustness of the periodic structure signal might influence translation and alternative splicing.

\section{Distribution of icSHAPE Reactivity Profiles In Vivo and In Vitro Across the CDS Regions and UTR Regions}

The average icSHAPE score of the transcripts in vivo and in vitro across the CDS regions and UTR regions to quantify the distribution of icSHAPE reactivity profiles in the ring and trophozoite stage development. Figure 3 shows that RNAs are more folded or crowded by a specific protein in vivo (low icSHAPE scores), and the extent of folding varies in different regions of RNAs in ring stage and trophozoite stage. In the ring stage, the 5' UTRs exhibited noticeable but partial unfolding, with the largest variation being observed between the CDS regions and the 3' UTRs. These three different regions of RNAs have nearly the same icSHAPE scores in vivo, but the CDS region has higher icSHAPE scores than the 5' UTR and 3' UTR when the RNA structure is modified in vitro. Then, we compared the average icSHAPE score collected from three 


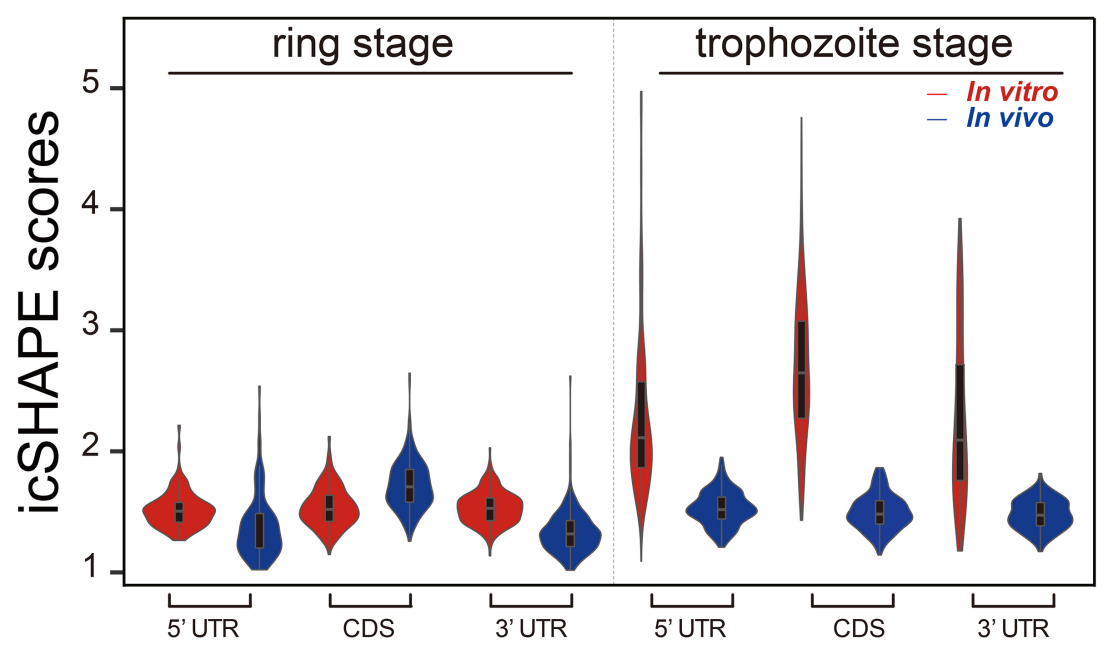

FIGURE 3 | icSHAPE reveals new features of mRNA secondary structures that located 5-UTR, CDS region and 3-UTR region in vivo and in vitro icSHAPE reveals new features of mRNA secondary structures that located 5-UTR, CDS region and 3-UTR region. The average icSHAPE score in vivo and in vitro across the CDS regions and UTR regions to quantify the distribution of icSHAPE reactivity profiles in the trophozoite stage development. The figure showed that RNAs are less folded in vivo (low icSHAPE scores), and the extent of unfolding varies in different regions of RNAs. The 5' UTRs exhibited noticeable but partial unfolding, with the largest variation compared to the CDS regions and 3' UTRs. Those three different regions of RNAs have nearly the same icSHAPE scores in vivo, but the CDS region have the highest icSHAPE scores than the 5' UTR and 3' UTR when the RNA structure were modified in vitro.

different regions of RNAs in trophozoite stage, the results also exhibited higher icSHAPE scores in vitro than that in vivo. Collectively, our data suggest that RNAs are more folded or crowded by a specific protein in vivo.

We examined the average icSHAPE score in vivo and in vitro across the CDS regions and UTR regions to quantify the distribution of icSHAPE reactivity profiles. Due to some nucleotides that were crowded in vivo and/or protein binding, or experimental limitations, we observed that icSHAPE scores from in vivo and in vitro are different. In our research, we found that RNAs are more folded or crowded in vivo (low icSHAPE scores), consistent with previous reports (Rouskin et al., 2014; Spitale et al., 2015), and the extent of unfolding was determined to vary in different regions of RNAs (Figures 4A, B). It has been revealed that the scale and distribution of RNA structural dynamics under in vitro conditions, which are refolded entirely by genome sequencing, are different from those under in vivo conditions, in which RNA molecular folding occurs depending on the context of the intracellular environment (Schroeder et al., 2002). Similar results were observed in metazoans (Li et al., 2012a) and humans (Wan et al., 2014), in which UTRs are, on average, more structured than coding regions. However, this global trend is different than that in yeast (Kertesz, 2010) and in E. coli (Del Campo et al., 2015), in which UTRs are less structured than CDSs.

\section{Structure Analysis Across the CDS Regions and UTR Regions In Vivo}

We performed structure analysis across the CDS regions and UTR regions in vivo. By PARS technology, Yue Wan et al. (Wan et al., 2014) were used to analyse more than 3,000 secondary structure human messenger RNAs, and found that it is more complicated and complex RNA secondary structures in the UTR regions than the CDS regions. Structures from the UTR, especially the 5' UTR, have an important role in the process of development due to their interaction with regulatory proteins. To explore this hypothesis in our experiment, we focused on two structured 5' UTRs and on the structured 3' UTR for more detailed functional analyses (Figures 4A, B). Figure 4 shows the icSHAPE signal in vivo from the ring stage and trophozoite stage libraries. We compared the average icSHAPE score collected from the four genomic features. The results exhibited significant differences from one region to another region in the RNA structure.

Metagene analysis of the transcripts, which aligned at their start and stop codons, shows that $P$. falciparum CDS regions have a propensity to form double-stranded structure (lower icSHAPE scores) to a level that is similar to the structural propensity of the 5'- and 3'-untranslated regions (UTRs). Additionally, our data indicated that lower icSHAPE scores in the sites from nt -25 to -21 in trophozoite stage than in the other sites of 5'UTR region of the transcriptome. This low icSHAPE scores means that those regions are more structured or protein binding sites of mRNA secondary structures. These positions may provide candidate sites for the functional conformation of mRNA, but require further investigation.

We next investigated whether a correlation is shared between intrinsic mRNA secondary structure propensity around the translation start/stop site and whether the efficiency of protein translation correlates with mRNA abundance. In Figures 4A, B, we also found that nt -1 to 4 and the last four base pairs of the CDS region of the transcriptome had significantly (Student's ttest, $\mathrm{P}<0.05$ ) higher icSHAPE scores than average, consistent with previous findings (Ding et al., 2014; Righetti et al., 2016). 


\section{A}
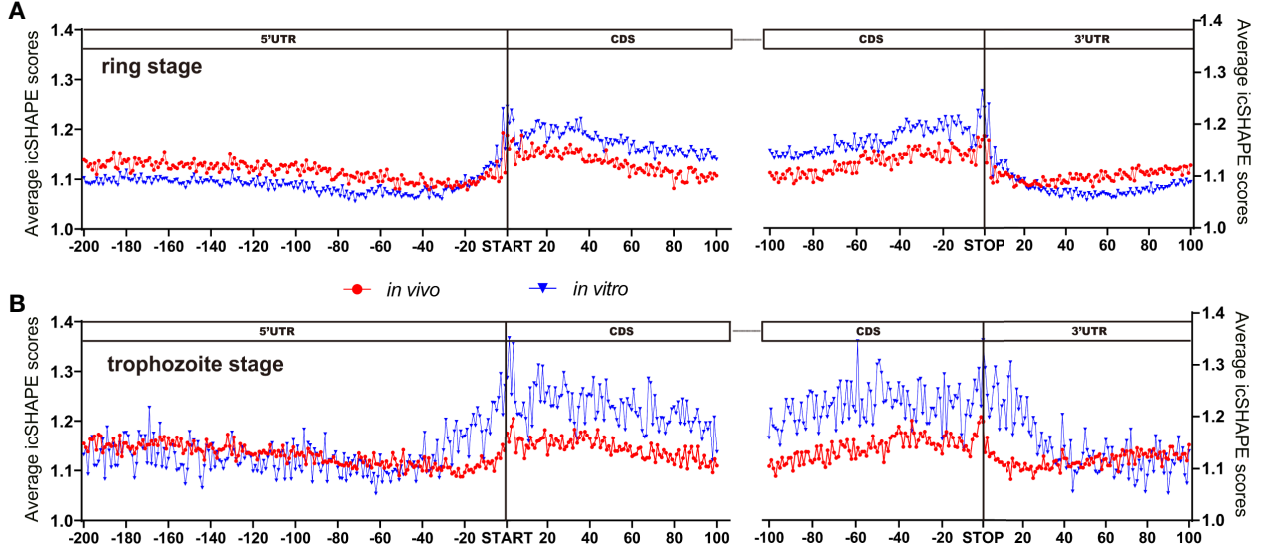

FIGURE 4 | icSHAPE reveals new features of mRNA secondary structures that located 5-UTR, CDS region and 3-UTR region. (Ring stage and trophozoite stage in vivo and in vitro compare) Average icSHAPE scores in selected regions of the whole expressed transcripts across the ring stage (A for ring stage and $\mathbf{B}$ for trophozoite stage) of parasites that have 5' and 3' UTR regions longer than $200 \mathrm{nt}$ 5' UTR region (200 nt upstream of the start codon); CDS initial region (100 nt downstream of the start codon); CDS final region (100 nt upstream of the stop codon); and 3' UTR region (100 nt downstream of the stop codon) are displayed. The start and stop codons of mRNA transcripts are indicated by grey bars; The first 4 nt immediately upstream of the start codon show significantly higher reactivity than the average icSHAPE scores across the first $100 \mathrm{nt}$ of the CDS with Significantly different (Student's t-test, $\mathrm{P}<0.05$ ).

Notably, the start and stop codons of each transcript exhibit higher icSHAPE scores, indicating reduced tendency for doublestranded conformation and easily increased accessibility for the expression of transcripts.

\section{mRNA Structure Correlates With mRNA Abundance in $P$. falciparum}

It is clear that mRNA structure is important for a variety of biological processes, including maintenance of RNA half-life and stability (Carrier and Keasling, 1997). We next asked whether the RNA secondary structure of the CDS correlates with mRNA abundance in the parasites. To quantify the transcriptome, we performed RNA-seq (Supplementary Table 6) with the parasites sample under the same condition as those used for treatment with $\mathrm{NAI}-\mathrm{N}_{3}$ which exhibited high reproducibility between biological replicates.

Comparison of the average icSHAPE score over the CDS revealed a very clear correlation with the mRNA abundance with this combined approach (Figure 5). A higher icSHAPE scores, those positions are treated as that they were more accessible to chemical modification, means that those regions are in the presence of a single strand. A lower icSHAPE scores, the positions are treated as that they were unaccessible to chemical modification, indicate the presence of a double strand or those regions are crowded by a specific protein. Our data in Figure 5 showed that the most abundant transcripts exhibited higher icSHAPE scores. Collectively, our data indicate that it's has unfolding of structured in those most abundant transcripts and open their double-strand with reduced tendency for doublestranded conformation in order to increase the accessibility for the following of ribosomal protein binding easily. In the other hand, due to some nucleotides which were crowded in vivo and/ or protein binding, they were not chemically reactive, and no complete coverage was be provided in our experiment for this long transcript, the Pearson correlations in our results seem not high but with reasonable. Therefore, we hypothesized that the RNA structure could regulate the activation/repression of gene expression in P. falciparum. Combined analysis of icSHAPE with transcriptome data demonstrated that stage-specific RNA structure plays an important role in the regulation of gene expression in $P$. falciparum.

\section{DISCUSSION}

In this study, to the best of our knowledge, we presented for the first global view of the genome-scale landscape of RNA secondary structures of the major human pathogen $P$. falciparum for all four bases in vivo and in vitro at two developmental stages at single-nucleotide resolution. The major strength of this scarcity of our RNA secondary structure was its potential use and important implications for understanding parasite gene expression and development. Recent studies have established RNA structure as a ubiquitous and fundamental regulator of gene expression (Mustoe et al., 2018) that can be used as an extremely specific and subtle mechanism for fine tuning a variety of cellular processes (Vandivier et al., 2016) within picoseconds to seconds (Ganser et al., 2019). On the other hand, Plasmodium parasites have 4 to 8 nucleus-encoded, structurally distinct, and differentially transcribed rRNA genes in different developmental stages (Gunderson et al., 1987; Qi et al., 2015). This finding also prompted us to identify the RNA secondary structure as an important factor that contributes to posttranslational control, especially in Plasmodium parasites. 

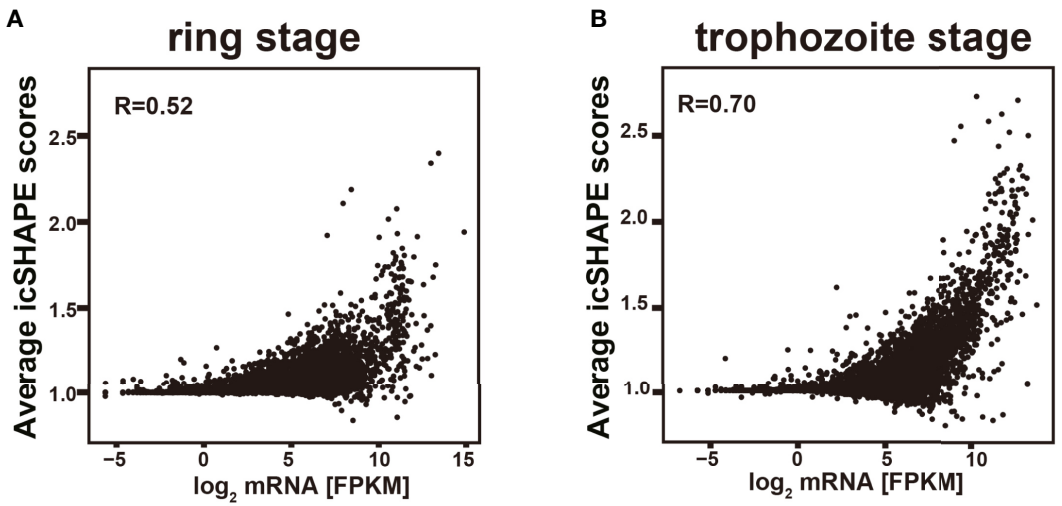

FIGURE 5 | Relationship of icSHAPE and expression abundant (FPKM) from RNA-seq analysis. Comparison of the average icSHAPE scores over the whole transcripts CDS regions revealed a clear correlation with the mRNA abundance. Dependence of the average icSHAPE scores on the mRNA abundance of the whole transcripts from ring stage at 37 degree $(\mathbf{A})$, and also the trophozoite stage at 37 degree $\mathbf{( B )}$. Pearson correlation coefficient (R) is showed in each panel.

Prior to NAI- $\mathrm{N}_{3}$ treatment, NAI- $\mathrm{N}_{3}$ was titred to single-hit kinetics in structure probing at the different temperatures to ensure that on average, any one copy of any RNA molecule was methylated only once. The major difference between the in vivo and in vitro conditions for SHAPE mapping is the cell walls and membranes encasing the transcriptomes of these organisms (Lee et al., 2017), especially the parasites that are located in the RBCs. For mapping RNA structures using either nuclease (RNase S1/ RNase V1) or DMS, NAI-N ${ }_{3}$ chemical probes, single-hit kinetics are one of the key factors (Kwok et al., 2015), because the over/no modification and/or cleavage of each site can cause conformational changes that lead to erroneous conclusions for specific structures. Moreover, the cell walls and cell membranes encasing the transcriptomes of different organisms are different, which makes the single-hit kinetics for diverse organisms different. Finally, we observed that modifying RNA inside parasite cells with $100 \mathrm{mM} \mathrm{NAI-N_{3 }}$ for 15 min yielded a goodquality RNA secondary structure at $37^{\circ} \mathrm{C}$. This method can also be used for other organisms, especially for organisms still without characterized RNA secondary structures.

With the development of high-throughput sequencing techniques over the past half-decade, methods for RNA structure probing have improved from single-gene analysis to whole-transcriptome interrogation. Therefore, we can produce a map of the 'RNA structurome' in a given organism to investigate the structural landscape of eukaryotic transcriptomes and highlight the relationship between some specific RNA structures and functions (Kwok et al., 2015). Chemical modification or RNase digestion are two usually powerful methods that have been used most extensively to map RNA structure in vivo and in vitro (Lucks et al., 2011; Mortimer et al., 2014; Wildauer et al., 2014; Lee et al., 2017). Recently, a new chemoaffinity structure probing methodology, icSHAPE (in vivo click selective 2-hydroxyl acylation and profiling experiment), using a novel bifunctional chemical probe, NAI- $\mathrm{N}_{3}$, for in vivo RNA structure profiling in the genome has become a well- established tool for the analysis of RNA structure, and labelling each accessible single-stranded nucleotide provides a higherresolution picture of the secondary structure of each transcript (Spitale et al., 2015).

Using the new chemoaffinity structure probing methodology icSHAPE, we generated a map of the 'RNA structurome' in Plasmodium, investigated the structural landscape of transcriptomes and highlighted the relationship between some specific RNA structures and their functions. Comparison of the static snapshots of our RNA structurome analysis in two developmental stages showed that the RNA structurome at two developmental stages possessed dynamic developmental responsive reorganization, long-range structures and higherorder architectures across the Plasmodium transcriptome. There was only 329 valid structural profiles for transcripts were obtained from in vitro $37^{\circ} \mathrm{C}$ treated polyA-selected RNA trophozoite stage libraries for the following reasons. First, the structural profiles that we define to valid structural profiles for transcripts are based on the transcripts which the reverse transcription stop coverage no less than 2 and background base density higher than 200. This is stricter than previously research. Second, the main reasons of less structural profiles from protein coding RNAs at the trophozoite stage compared to the ring stage is the low number of raw reads in trophozoite stage. Lower number of raw reads, less structural profiles. For the current study, we would like to focus on resolution and genomewide RNA secondary structural in $P$. falciparum and the connection between RNA secondary structure and mRNA abundance during the complex biological program of $P$. falciparum. We therefore have not performed additional in vitro icSHAPE experiments (trophozoite stage) even with low coverage. In vitro structural profiles from protein coding RNAs at the trophozoite stage is for further investigation.

Although there is significant global correspondence between our icSHAPE-modified RNA structures and known structures, there are still some differences between icSHAPE and known 
structures; one reason for the differences is the noise in our approach, and the other reason is the known inaccuracies of folding algorithms (Kertesz, 2010). Therefore, these NAI-N $3^{-}$ independent stops occurred at known endogenously modified rRNA nucleotides; thus, we conclude that icSHAPE can successfully and accurately map chemically modified nucleotides on large RNAs on a genome-wide basis from Plasmodium parasites. Due to some nucleotides that were crowded in vivo and/or protein binding or experimental limitations, they were not chemically reactive, and no complete coverage was provided in our single experiment for this long transcript. Our partial but accurate RNA secondary structure can provide important information at some vital position of each transcript. Our icSHAPE results are strongly correlated with the results from our capillary electrophoresis-based probing method, and it can be used for accurate mapping of the RNA secondary structure. Our in vivo icSHAPE data are in excellent agreement with known RNA structures.

Although icSHAPE RNA secondary data were obtained from two stages of the Plasmodium and many genes expressed in other asexual developmental stages or mosquito/liver stages could not be obtained here, this study still provides important information on RNA secondary structure and the changes in structures between the two stages. Our study may have had several limitations. The depth was not enough to cover all of the whole transcripts, and larger studies with longer follow-up are needed to obtain RNA secondary structure information from the very low-level transcripts. Recently, a method named hiCLIP (Sugimoto et al., 2015) (RNA hybrid and individual nucleotide resolution ultraviolet crosslinking and immunoprecipitation), uses crosslinking and proximity ligation to determine a special RNA structures bound by special double-stranded RNA-binding proteins. This method may be a good choice for the determination of low-level transcripts or transcripts with complex RNA duplexes.

At present, researchers have found that mutations in many RNA helicases may lead to cancers (Fuller-Pace, 2013; Sullenger and Nair, 2016). Therefore, the application of the RNA secondary structure determination methods to these diverse problems and our parasites field will help us to identify some basic mechanisms of gene expression and potential therapeutic opportunities for treating diseases and parasites. Our findings also open avenues for the influence of specific RNA structural features on gene expression. It has yielded insights into the regulatory roles of RNA secondary structure in many organisms and cellular conditions (Wan et al., 2011; Strobel et al., 2018; Aw et al., 2020).

The development of Plasmodium parasites requires two hosts and completes 11 different stages. Therefore, there is a quick efficient response to various environmental changes. In the future, in vivo RNA structure studies under different stress conditions, such as temperature (Fang and McCutchan, 2002), the host body of nutrient supply (Mancio-Silva et al., 2017), amino acid (Babbitt et al., 2012) and glucose concentration in the blood (Fang et al., 2004), could provide clues about the structural characteristics of these dynamic mRNAs and their regulatory roles (Kwok et al., 2015).
In summary, our study presents the first icSHAPE-enabled transcriptome-wide secondary structure map and adds to the accumulating evidence that there is a critical connection between RNA structure and the complex biological programme of $P$. falciparum. Our results showed that the average icSHAPE score over the CDS revealed a very clearly correlation with the mRNA abundance. This means that stage specific RNA structure also play an important role in the regulation of gene expression in P. falciparum.

\section{DATA AVAILABILITY STATEMENT}

The datasets presented in this study can be found in online repositories. The names of the repository/repositories and accession number(s) can be found in the National Center for Biotechnology Information (NCBI) Sequence Read Archive under the BioProject ID PRJNA625343.

\section{AUTHOR CONTRIBUTIONS}

YQ designed and performed the experiments, analysed data, prepared figures, writing, review, and editing original draft. YZ and GZ designed and performed the experiments, analysed data, and prepared figures. BC and MZ prepared figures and performed analysis. JL, TP, and $\mathrm{JH}$ review and editing. XW designed and supervised the study. All authors performed data quantification, discussed the results, and commented on the manuscript. All authors contributed to the article and approved the submitted version.

\section{FUNDING}

This research was funded by Natural Science Foundation of China (Grant number: 81902087), Guangdong Natural Science Fund Project (Grant number: 2017A030310535), and China postdoctoral Science Foundation (Grant number: 2016M592474).

\section{ACKNOWLEDGMENTS}

The authors would like to thank Jianbing $\mathrm{Mu}$ at National Institutes of Health, USA for his discussion and technical assistance.

\section{SUPPLEMENTARY MATERIAL}

The Supplementary Material for this article can be found online at: https:/www.frontiersin.org/articles/10.3389/fcimb.2021.673966/ full\#supplementary-material

Supplementary Figure 1 | Determination of the conditions for single-hit kinetics Determination of the conditions for single-hit kinetics. Time course of $\mathrm{NAI}-\mathrm{N}_{3}$ modification and capillary electrophoresis data accurately maps to known 
structures. Lysis parasites were NAl- $\mathrm{N}_{3}$ treated for different durations (5 min, 10min, $15 \mathrm{~min}$ and $30 \mathrm{~min}$ ) with the final NAl- $\mathrm{N}_{3}$ concentration was $100 \mathrm{mM}$. The $18 \mathrm{~S} \mathrm{A-}$ type rRNA NAI- $\mathrm{N}_{3}$ modification read-out was assessed by capillary electrophoresisbased probing, which was done here near the 5' end (200bp-250bp, the region between two orange peaks). " 5 min", "10min", "15 min" and "30 min" show the parasite lysis were incubated with $100 \mathrm{mM} \mathrm{NAI}-\mathrm{N}_{3}$ for $5 \mathrm{~min}, 10 \mathrm{~min}, 15 \mathrm{~min}$ and $30 \mathrm{~min}$ at $37^{\circ} \mathrm{C}$ water bath respectively. "Control" show the parasite lysis were incubated with DMSO for 30 min at $37^{\circ} \mathrm{C}$ water bath. The red-blue stripe below "15 min" indicate the double-(red) or single-stranded (blue) RNA in this region.

Supplementary Figure 2 | Determination of fragmentation time by primer extension electropherograms Determination of fragmentation time by primer extension electropherograms. Appropriate sizes of fragments are need for efficient obtain modification sites via RT-PCR and sequencing. Short RNAs, $\sim 100 \mathrm{nt}$, are the most strategies that have been optimized to achieve single-hit kinetics of chemical modification. "1 min", "2min", "3 min" and " 4 min" show the total RNA were fragmented for $1 \mathrm{~min}, 2 \mathrm{~min}, 3 \mathrm{~min}$ and $4 \mathrm{~min}$ at $70^{\circ} \mathrm{C} \mathrm{PCR}$ machine respectively. "Control" show the total RNA were no fragmented. The orange pink is GeneScan 500LIZ size standard, provides 5 single-stranded labeled fragments of: 50, 75, 100, 139 and 150 nucleotides.

\section{REFERENCES}

Aw, J. G. A., Lim, S. W., Wang, J. X., Lambert, F. R. P., Tan, W. T., Shen, Y., et al. (2020). Determination of Isoform-Specific RNA Structure With Nanopore Long Reads. Nat. Biotechnol. 39 (3), 336-346. doi: 10.1038/s41587-0200712-z

Babbitt, S. E., Altenhofen, L., Cobbold, S. A., Istvan, E. S., Fennell, C., Doerig, C., et al. (2012). Plasmodium falciparum Responds to Amino Acid Starvation by Entering Into a Hibernatory State. Proc. Natl. Acad. Sci. U. S. A. 109 (47), E3278-E3287. doi: 10.1073/pnas.1209823109

Bevilacqua, P. C., Ritchey, L. E., Su, Z., and Assmann, S. M. (2016). Genome-Wide Analysis of RNA Secondary Structure. Annu. Rev. Genet. 50 (1), 235-266. doi: 10.1146/annurev-genet-120215-035034

Bolger, A. M., Lohse, M., and Usadel, B. (2014). Trimmomatic: A Flexible Trimmer for Illumina Sequence Data. Bioinformatics 30 (15), 2114-2120. doi: 10.1093/bioinformatics/btu170

Cannone, J. J., Subramanian, S., Schnare, M. N., Collett, J. R., D’Souza, L. M., Du, Y., et al. (2002). The Comparative RNA Web (CRW) Site: An Online Database of Comparative Sequence and Structure Information for Ribosomal, Intron, and Other Rnas. BMC Bioinf. 3, 2. doi: 10.1186/1471-2105-3-2

Capela, R., Moreira, R., and Lopes, F. (2019). An Overview of Drug Resistance in Protozoal Diseases. Int. J. Mol. Sci. 20 (22), 5748. doi: 10.3390/ijms20225748

Carrier, T. A., and Keasling, J. D. (1997). Controlling Messenger RNA Stability in Bacteria: Strategies for Engineering Gene Expression. Biotechnol. Prog. 13 (6), 699-708. doi: 10.1021/bp970095h

Choi, L., Pryce, J., and Garner, P. (2019). Indoor Residual Spraying for Preventing Malaria in Communities Using Insecticide-Treated Nets. Cochrane Database Syst. Rev. 5:CD012688. doi: 10.1002/14651858.CD012688.pub2

Del Campo, C., Bartholomaus, A., Fedyunin, I., and Ignatova, Z. (2015). Secondary Structure Across the Bacterial Transcriptome Reveals Versatile Roles in mRNA Regulation and Function. PLoS Genet. 11 (10), e1005613. doi: 10.1371/ journal.pgen.1005613

Ding, Y., Kwok, C. K., Tang, Y., Bevilacqua, P. C., and Assmann, S. M. (2015). Genome-Wide Profiling of In Vivo RNA Structure At Single-Nucleotide Resolution Using Structure-Seq. Nat. Protoc. 10 (7), 1050-1066. doi: 10.1038/nprot.2015.064

Ding, Y., Tang, Y., Kwok, C. K., Zhang, Y., Bevilacqua, P. C., and Assmann, S. M. (2014). In Vivo Genome-Wide Profiling of RNA Secondary Structure Reveals Novel Regulatory Features. Nature 505 (7485), 696-700. doi: 10.1038/ nature12756

Fang, J., and McCutchan, T. F. (2002). Thermoregulation in a Parasite's Life Cycle. Nature 418 (6899):742. doi: 10.1038/418742a

Fang, J., Sullivan, M., and McCutchan, T. F. (2004). The Effects of Glucose Concentration on the Reciprocal Regulation of rRNA Promoters in Plasmodium falciparum. J. Biol. Chem. 279 (1), 720-725. doi: 10.1074/ jbc.M308284200
Supplementary Figure 3 | Secondary structure of Plasmodium falciparum $18 \mathrm{~S}$ small subunit ribosomal RNA (blood stage) in vivo NAI- $\mathrm{N}_{3}$ modification identified by icSHAPE.

Supplementary Figure 4 | Overview of the icSHAPE approach in our experiment Overview of the icSHAPE approach used for temperature-responsive in vivo and in vitro RNA structurome of Plasmodium falciparum in our experiment. P. falciparum was cultured in human $\mathrm{O}+$ erythrocytes at $5 \%$ hematocrit at $37^{\circ} \mathrm{C}$. Cultures were synchronized twice at ring stage with $5 \%$ D-sorbitol treatments performed 8 hours apart. We performed to culture parasites at 48/72 (ring stage/trophozoite stage) hours after the first synchronization experiment. Then parasites were lysis by $1 \%$ saponin, following by RNA structure modification by NAI- $\mathrm{N}_{3}$ or extract total RNA from parasite directly. During NAI- $\mathrm{N}_{3}$ modification, we set a DMSO-treated negative control sample for that the DMSO sample can provides an 'input' sample. In vitro RNA structure experiments were done by treating the total RNA from DMSOtreated sample with NAI- $\mathrm{N}_{3}$. After library preparation and Illumina sequencing, the count of mapping reads from sequencing generated $\mathrm{NAl}-\mathrm{N}_{3}$ in vitro, $\mathrm{NAI}-\mathrm{N}_{3}$ in vivo, and DMSO-treated control profiles. Finally, visualization the RNA secondary structure at difference stages and find the relationship between RNA secondary structure and gene expression.

Flynn, R. A., Zhang, Q. C., Spitale, R. C., Lee, B., Mumbach, M. R., and Chang, H. Y (2016). Transcriptome-Wide Interrogation of RNA Secondary Structure in Living Cells With Icshape. Nat. Protoc. 11 (2), 273-290. doi: 10.1038/nprot.2016.011

Fuller-Pace, F. V. (2013). DEAD Box RNA Helicase Functions in Cancer. RNA Biol. 10 (1), 121-132. doi: 10.4161/rna.23312

Ganser, L. R., Kelly, M. L., Herschlag, D., and Al-Hashimi, H. M. (2019). The Roles of Structural Dynamics in the Cellular Functions of Rnas. Nat. Rev. Mol. Cell Biol. 20 (8), 474-489. doi: 10.1038/s41580-019-0136-0

Gunderson, J. H., Sogin, M. L., Wollett, G., Hollingdale, M., de la Cruz, V. F., Waters, A. P., et al. (1987). Structurally Distinct, Stage-Specific Ribosomes Occur in Plasmodium. Science 238 (4829), 933-937. doi: 10.1126/science.3672135

Incarnato, D., Neri, F., Anselmi, F., and Oliviero, S. (2014). Genome-Wide Profiling of Mouse RNA Secondary Structures Reveals Key Features of the Mammalian Transcriptome. Genome Biol. 15, 491. doi: 10.1186/s13059-014-0491-2

Jin, Y., Yang, Y., and Zhang, P. (2011). New Insights Into RNA Secondary Structure in the Alternative Splicing of Pre-Mrnas. RNA Biol. 8 (3), 450-457. doi: 10.4161/rna.8.3.15388

Kaushik, K., Sivadas, A., Vellarikkal, S. K., Verma, A., Jayarajan, R., Pandey, S., et al. (2018). RNA Secondary Structure Profiling in Zebrafish Reveals Unique Regulatory Features. BMC Genomics 19 (1), 147. doi: 10.1186/s12864-018-4497-0

Kertesz, M. (2010). Genome-Wide Measurement of RNA Secondary Structure in Yeast. Nature 467, 103-107. doi: 10.1038/nature09322

Kwok, C. K., Tang, Y., Assmann, S. M., and Bevilacqua, P. C. (2015). The RNA Structurome: Transcriptome-Wide Structure Probing With Next-Generation Sequencing. Trends Biochem. Sci. 40 (4), 221-232. doi: 10.1016/j.tibs.2015.02.005

Lambros, C., and Vanderberg, J. P. (1979). Synchronization of Plasmodium falciparum Erythrocytic Stages in Culture. J. Parasitol. 65 (3), 418-420.

Langmead, B., Trapnell, C., Pop, M., and Salzberg, S. L. (2009). Ultrafast and Memory-Efficient Alignment of Short DNA Sequences to the Human Genome. Genome Biol. 10 (3), R25. doi: 10.1186/gb-2009-10-3-r25

Lee, B., Flynn, R. A., Kadina, A., Guo, J. K., Kool, E. T., and Chang, H. Y. (2017). Comparison of SHAPE Reagents for Mapping RNA Structures Inside Living Cells. RNA 23 (2), 169-174. doi: 10.1261/rna.058784.116

Li, F., Zheng, Q., Ryvkin, P., Dragomir, I., Desai, Y., Aiyer, S., et al. (2012a). Global Analysis of RNA Secondary Structure in Two Metazoans. Cell Rep. 1 (1), 6982. doi: 10.1016/j.celrep.2011.10.002

Li, F., Zheng, Q., Vandivier, L. E., Willmann, M. R., Chen, Y., and Gregory, B. D. (2012b). Regulatory Impact of RNA Secondary Structure Across the Arabidopsis Transcriptome. Plant Cell 24 (11), 4346-4359. doi: 10.1105/tpc.112.104232

Lucks, J. B., Mortimer, S. A., Trapnell, C., Luo, S. J., Aviran, S., Schroth, G. P., et al. (2011). Multiplexed RNA Structure Characterization With Selective 2 '-Hydroxyl Acylation Analyzed by Primer Extension Sequencing (SHAPE-Seq). Proc. Natl. Acad. Sci. U. S. A. 108 (27), 11063-11068. doi: 10.1073/pnas.1106501108

Mancio-Silva, L., Slavic, K., Grilo Ruivo, M. T., Grosso, A. R., Modrzynska, K. K., Vera, I. M., et al. (2017). Nutrient Sensing Modulates Malaria Parasite Virulence. Nature 547 (7662), 213-216. doi: 10.1038/nature23009 
Merino, E. J., Wilkinson, K. A., Coughlan, J. L., and Weeks, K. M. (2005). RNA Structure Analysis At Single Nucleotide Resolution by Selective 2'-Hydroxyl Acylation and Primer Extension (SHAPE). J. Am. Chem. Soc. 127 (12), 42234231. doi: $10.1021 /$ ja043822v

Mignone, F., Gissi, C., Liuni, S., and Pesole, G. (2002). Untranslated Regions of Mrnas. Genome Biol. 3 (3), REVIEWS0004. doi: 10.1186/gb-2002-3-3-reviews0004

Mortimer, S. A., Kidwell, M. A., and Doudna, J. A. (2014). Insights Into RNA Structure and Function From Genome-Wide Studies. Nat. Rev. Genet. 15 (7), 469-479. doi: 10.1038/nrg3681

Mustoe, A. M., Busan, S., Rice, G. M., Hajdin, C. E., Peterson, B. K., Ruda, V. M., et al. (2018). Pervasive Regulatory Functions of mRNA Structure Revealed by High-Resolution Shape Probing. Cell 173 (1), 181-195.e118. doi: 10.1016/ j.cell.2018.02.034

Qi, Y., Zhu, F., Eastman, R. T., Fu, Y., Zilversmit, M., Pattaradilokrat, S., et al. (2015). Regulation of Plasmodium yoelii Oocyst Development by Strain- and Stage-Specific Small-Subunit Rrna. MBio 6 (2), e00117. doi: 10.1128/ mBio.00117-15

Quinlan, A. R., and Hall, I. M. (2010). Bedtools: A Flexible Suite of Utilities for Comparing Genomic Features. Bioinformatics 26 (6), 841-842. doi: 10.1093/ bioinformatics/btq033

Righetti, F., Nuss, A. M., Twittenhoff, C., Beele, S., Urban, K., Will, S., et al. (2016). Temperature-Responsive In Vitro RNA Structurome of Yersinia Pseudotuberculosis. Proc. Natl. Acad. Sci. U. S. A. 113 (26), 7237-7242. doi: 10.1073/pnas.1523004113

Rouskin, S., Zubradt, M., Washietl, S., Kellis, M., and Weissman, J. S. (2014). Genome-Wide Probing of RNA Structure Reveals Active Unfolding of mRNA Structures In Vivo. Nature 505 (7485), 701-705. doi: 10.1038/nature12894

Schroeder, R., Grossberger, R., Pichler, A., and Waldsich, C. (2002). RNA Folding In Vivo. Curr. Opin. Struct. Biol. 12 (3), 296-300. doi: 10.1016/s0959-440x(02)00325-1

Spitale, R. C., Flynn, R. A., Zhang, Q. C., Crisalli, P., Lee, B., Jung, J. W., et al. (2015). Structural Imprints In Vivo Decode RNA Regulatory Mechanisms. Nature 519 (7544), 486-490. doi: 10.1038/nature14263

Strobel, E. J., Yu, A. M., and Lucks, J. B. (2018). High-Throughput Determination of RNA Structures. Nat. Rev. Genet. 19 (10), 615-634. doi: 10.1038/s41576-018-0034-x

Sugimoto, Y., Vigilante, A., Darbo, E., Zirra, A., Militti, C., D’Ambrogio, A., et al. (2015). hiCLIP Reveals the In Vivo Atlas of mRNA Secondary Structures Recognized by Staufen 1. Nature 519 (7544), 491-494. doi: 10.1038/nature14280

Sullenger, B. A., and Nair, S. (2016). From the RNA World to the Clinic. Science 352 (6292), 1417-1420. doi: 10.1126/science.aad8709

Su, Z., Tang, Y., Ritchey, L. E., Tack, D. C., Zhu, M., Bevilacqua, P. C., et al. (2018). Genome-Wide RNA Structurome Reprogramming by Acute Heat Shock Globally Regulates mRNA Abundance. Proc. Natl. Acad. Sci. U. S. A. 115 (48), 12170-12175. doi: 10.1073/pnas.1807988115
Talkish, J., May, G., Lin, Y., Woolford, J. L. Jr., and McManus, C. J. (2014). ModSeq: High-Throughput Sequencing for Chemical Probing of RNA Structure. RNA 20 (5), 713-720. doi: 10.1261/rna.042218.113

Trager, W., and Jensen, J. B. (1976). Human Malaria Parasites in Continuous Culture. Science 193 (4254), 673-675. doi: 10.1126/science.781840

Vandivier, L. E., Anderson, S. J., Foley, S. W., and Gregory, B. D. (2016). The Conservation and Function of RNA Secondary Structure in Plants. Annu. Rev. Plant Biol. 67, 463-488. doi: 10.1146/annurev-arplant-043015-111754

Vasa, S. M., Guex, N., Wilkinson, K. A., Weeks, K. M., and Giddings, M. C. (2008). Shapefinder: A Software System for High-Throughput Quantitative Analysis of Nucleic Acid Reactivity Information Resolved by Capillary Electrophoresis. Rna-a Publ. RNA Soc. 14 (10), 1979-1990. doi: 10.1261/rna.1166808

Wan, Y., Kertesz, M., Spitale, R. C., Segal, E., and Chang, H. Y. (2011) Understanding the Transcriptome Through RNA Structure. Nat. Rev. Genet. 12 (9), 641-655. doi: 10.1038/nrg3049

Wan, Y., Qu, K., Zhang, Q. C., Flynn, R. A., Manor, O., Ouyang, Z., et al. (2014). Landscape and Variation of RNA Secondary Structure Across the Human Transcriptome. Nature 505 (7485), 706-709. doi: 10.1038/nature12946

Wildauer, M., Zemora, G., Liebeg, A., Heisig, V., and Waldsich, C. (2014). Chemical Probing of RNA in Living Cells. Methods Mol. Biol. 1086, 159176. doi: 10.1007/978-1-62703-667-2_9

Wong, W., Bai, X. C., Brown, A., Fernandez, I. S., Hanssen, E., Condron, M., et al. (2014). Cryo-EM Structure of the Plasmodium falciparum 80S Ribosome Bound to the Anti-Protozoan Drug Emetine. Elife 3, e03080. doi: 10.7554/ eLife.03080

World Health Organization (2020). World Malaria Report 2020 (Geneva: WHO Press).

Zheng, Q., Ryvkin, P., Li, F., Dragomir, I., Valladares, O., Yang, J., et al. (2010). Genome-Wide Double-Stranded Rna Sequencing Reveals the Functional Significance of Base-Paired RNAs in Arabidopsis. PLoS Genet. 6 (9), e1001141. doi: 10.1371/journal.pgen.1001141

Conflict of Interest: The authors declare that the research was conducted in the absence of any commercial or financial relationships that could be construed as a potential conflict of interest.

Copyright (c) 2021 Qi, Zhang, Zheng, Chen, Zhang, Li, Peng, Huang and Wang. This is an open-access article distributed under the terms of the Creative Commons Attribution License (CC BY). The use, distribution or reproduction in other forums is permitted, provided the original author(s) and the copyright owner(s) are credited and that the original publication in this journal is cited, in accordance with accepted academic practice. No use, distribution or reproduction is permitted which does not comply with these terms. 